\title{
TRANSCENDENCE MEASURES FOR EXPONENTIALS AND LOGARITHMS
}

\author{
MICHEL WALDSCHMIDT \\ Dedicated to K. Mahler on his 75th birthday
}

(Received 2 January 1978)

Communicated by J. H. Coates

\begin{abstract}
In the present paper, we derive transcendence measures for the numbers $\log \alpha, e^{\beta}$, $\alpha^{\beta}$, $\left(\log \alpha_{1}\right) /\left(\log \alpha_{2}\right)$ from a previous lower bound of ours on linear forms in the logarithms of algebraic numbers.
\end{abstract}

Subject classification (Amer. Math Soc. (MOS) 1970): 10 F 05, 10 F 35

\section{Introduction}

A great deal of work has already been done on finding transcendence measures for the numbers listed above; see, for example, the contributions of Mahler [Ma 1, 2, 3, 5], Gelfond [G] and Feldman [F 1, 2, 3, 4]. A systematic study of this subject is given in Cijsouw's thesis [Ci 1], and the sharpest results to date are due mainly to Cijsouw [Ci 1, 2, 3, 4]. However, until now, special arguments were needed for each given class of numbers. The novelty of the present paper is to give a single uniform proof of all these results, by using a lower bound for linear forms in the logarithms of algebraic numbers [W].

Our main results are summarized by the following diagrams. The notation is as follows. Let $\varphi(X, Y)$ be a real-valued function which is defined for $X \geqslant 1$ and $Y \geqslant \log 16$ (this is for convenience only). If $\omega$ is a transcendental number, we say that $\varphi$ is a transcendence measure for $\omega$ if $\log |P(\omega)| \geqslant-\varphi(N, \log H)$ for all nontrivial polynomials $P$ in $\mathbf{Z}[X]$ with degree at most $N$ and height (in the usual sense) at most $H$. In addition, we say that a real number $\tau \geqslant 2$ is a transcendence type 
for $\omega$ if there exists a constant $c(\omega, \tau)>0$ such that $c(\omega, \tau)(\log H+N)^{\tau}$ is a transcendence measure for $\omega$. Finally, for real $x$, we write $\log _{+} x=\log (\max (1, x))$.

In Fig. 1 we give the consequences of the lower bound of $[\mathrm{W}]$ for the transcendence measures of the numbers $\pi, \log \alpha, e^{\beta}$ and $\left(\log \alpha_{1}\right) /\left(\log \alpha_{2}\right)$, when $\alpha, \beta, \alpha_{1}, \alpha_{2}$ are non-zero algebraic numbers with $\log \alpha \neq 0$ and $\left(\log \alpha_{1}\right) /\left(\log \alpha_{2}\right)$ irrational. Moreover, we give a result concerning $\alpha^{\beta}$ (with $\alpha$ and $\beta$ algebraic, $\alpha \neq 0, \log \alpha \neq 0$ and $\beta$ irrational) which is obtained by combining our lower bound with a result of Choodnovsky [Ch].

\begin{tabular}{llc}
\hline \multicolumn{1}{c}{ Number } & \multicolumn{1}{c}{ Transcendence measure } & Type $\leqslant$ \\
\hline$\pi$ & $2^{40} N(\log H+N \log N)(1+\log N)$ & $2+\varepsilon$ \\
$\log \alpha$ & $C_{1} N^{2}(\log H+N \log N)(1+\log N)^{-1}$ & 3 \\
$e^{\beta}$ & $C_{2} N^{2}(\log H+\log N)(\log \log H+\log N)^{2}$ & 3 \\
& $\quad \times(\log \log H+\log \log N)^{-2}$ & 4 \\
$\left(\log \alpha_{1}\right) /\left(\log \alpha_{2}\right)$ & $C_{3} N^{3}(\log H+N \log N)(1+\log N)^{-2}$ & 4 \\
$\alpha^{\beta}$ & $C_{4} N^{3}(\log H+\log N)(\log \log H+\log N)(1+\log N)^{-2}$ & 4 \\
\hline
\end{tabular}

FIG. 1. Transcendence measures for classical numbers.

We are interested with transcendence measure which are explicit in $N$ and $H$ (some older papers are better when $H$ is large with respect to $N$ ). The estimate concerning $\pi$ is due to Fel'dman and Cijsouw; the result concerning $\log \alpha$ is due to Cijsouw. All the other results improve earlier known transcendence measures with respect to the degree $N$.

The number $e^{\pi}$ is worthy of special consideration (Fig. 2). The transcendence measure which is provided by our linear form improves earlier results in the case of large height. In [Ch], Choodnovsky announces a result which is much more precise when the degree is very large. In the middle case, a claim of the "Stellingen" of Cijsouw's thesis [Ci 1] leads to a still better result. From Cijsouw's result it follows that $e^{\pi}$ has a transcendence type at most 3 .

$\begin{array}{lll}\log H \geqslant N(\log N)^{3} & 2^{62} N^{2}(\log H)(\log \log H)(1+\log N) & \\ N(\log N)^{3} \geqslant \log H \geqslant N(\log N)^{-1} & C_{5} N(\log H+N)^{2}(\log \log H)^{-1} & \text { (Cijsouw) } \\ N(\log N)^{-1} \geqslant \log H & C_{6} N(\log H+\log N)^{2}(\log N) & \text { (Choodnovsky) }\end{array}$

Fig. 2. Transcendence measures for $e^{\pi}$.

Finally in Figure 3 (see p. 447) we give four general results which are consequences of our estimate [W], and which in fact contain several of the abovementioned results (see Theorems 5.1 and 5.3 below). 
The idea of deriving transcendence measures from a lower bound for linear forms in logarithms was used already in [M-W], and even earlier by Baker to give an irrationality measure for the number $e^{\pi}$ (cf. [B] Chap. 3).

In a subsequent paper we will consider simultaneous approximations and improve several results of $[\mathrm{C}-\mathrm{W}]$ and $[\mathrm{M}-\mathrm{W}]$.

\begin{tabular}{lcc}
\hline \multicolumn{1}{c}{ Number } & Transcendence measure & Type $\leqslant$ \\
\hline$e^{\beta_{0}} \alpha_{1}^{\beta_{1}} \ldots \alpha_{m}^{\beta_{m}}$ & $C_{7} N^{m+2}(\log H+\log N)$ & $m+3$ \\
& $\times(\log \log H+\log N)^{m+3}$ & \\
& $\quad \times(\log \log H)^{-m-2}(1+\log N)^{-m-1}$ & \\
$e^{\beta_{0}} \alpha_{1}^{\beta_{1}} \ldots \alpha_{m}^{\beta_{m}} e^{\gamma t \pi}$ & $C_{8} N^{m+2}(\log H+\log N)$ & $m+3+\varepsilon$ \\
& $\times(\log \log H+\log N)(1+\log N)$ & \\
$\frac{\beta_{0}+\beta_{1} \log \alpha_{1}+\ldots+\beta_{s} \log \alpha_{s}}{\beta_{0}^{\prime}+\beta_{1}^{\prime} \log \alpha_{1}^{\prime}+\ldots+\beta_{t}^{\prime} \log \alpha_{t}^{\prime}}$ & $C_{9} N^{s+t+1}(\log H+N \log N)$ & $s+t+2$ \\
$\frac{\beta_{0}+\beta_{1} \log \alpha_{1}+\ldots+\beta_{s} \log \alpha_{g}+\gamma i \pi}{\beta_{0}^{\prime}+\beta_{1}^{\prime} \log \alpha_{1}^{\prime}+\ldots+\beta_{t}^{\prime} \log \alpha_{t}^{\prime}+\gamma^{\prime} i \pi}$ & $\times(1+\log N)^{-s-t}$ & \\
\hline
\end{tabular}

Fig. 3. Four general results.

This paper has been written at the University of New South Wales (Australia). The author wishes to thank his colleagues from Kensington, and especially Alf van der Poorten, for their hospitality.

\section{Notations and preliminary lemmas}

Let $\boldsymbol{P}=a_{0} X^{N}+\ldots+a_{N} \in \mathbf{C}[X]$ be any non-zero polynomial with complex coefficients, with $a_{0} \neq 0$. Denote by $N(P)=N$ its degree, by

$$
H(P)=\max _{0 \leqslant j \leqslant N}\left|a_{j}\right|
$$

its "usual height", by

$$
L(P)=\sum_{j=0}^{N}\left|a_{j}\right|
$$

its "length", and by

$$
M(P)=\exp \int_{0}^{1} \log \left|P\left(e^{2 i \pi t}\right)\right| d t
$$

its "measure". If $\alpha_{1}, \ldots, \alpha_{N}$ are the roots of $P$ (according to multiplicity), then by Jensen's formula we have [Ma 4]:

$$
M(P)=\left|a_{0}\right| \prod_{j=1}^{N} \max \left\{1,\left|\alpha_{j}\right|\right\}
$$


This measure has been introduced by Mahler who used the trivial formula

$$
M\left(P_{1} P_{2}\right)=M\left(P_{1}\right) M\left(P_{2}\right) \text { for } P_{1}, P_{2} \in \mathrm{C}[X]
$$

to deduce non-trivial relations between $H\left(P_{1}, P_{2}\right)$ and $H\left(P_{1}\right) \cdot H\left(P_{2}\right)$. Here we need only the inequality

$$
\log M(P) \leqslant \log H(P)+\log N(P),
$$

(see [Ma 4]), which can be deduced from

where

$$
M(P) \leqslant\|P\| \leqslant(N+1)^{\frac{1}{2}} H(P)
$$

$$
\|P\|=\left(\sum_{j=0}^{N}\left|a_{j}^{\prime}\right|^{2}\right)^{\frac{1}{2}}
$$

(see [M-W] Lemma 1).

The most important properties of Mahler's measure $M$ concern algebraic numbers. Let $\alpha$ be an algebraic number, and $P \in \mathbf{Z}[X]$ be its minimal polynomial. We define the degree, usual height, length and measure of $\alpha$ by

$$
N(\alpha)=N(P), \quad H(\alpha)=H(P), \quad L(\alpha)=L(P), \quad M(\alpha)=M(P) .
$$

From (2.1) we get

$$
\log M(\alpha) \leqslant \log H(\alpha)+\log N(\alpha) .
$$

The multiplicative property of the measure of polynomials is no longer true for the measure of algebraic numbers (because the minimal polynomial of the product of algebraic numbers is usually different from the product of the minimal polynomials). However, if we define the "absolute logarithmic height" of $\alpha$ by

$$
h(\alpha)=\frac{1}{N(\alpha)} \log M(\alpha),
$$

then $h\left(\alpha_{1} \alpha_{2}\right) \leqslant h\left(\alpha_{1}\right)+h\left(\alpha_{2}\right)$ and $h\left(\alpha^{m}\right)=m h(\alpha)$ for all algebraic numbers $\alpha_{1}, \alpha_{2}, \alpha$ and any non-zero rational integer $m$. These properties follow from the very useful connection between $h$ and the height on projective spaces of Neron and Lang (cf. [W]). It follows that the absolute logarithmic height is far easier to handle for transcendence proofs than other "size" functions.

We have followed the tradition in Section 1 by defining transcendence measure in terms of the usual height. But it will be much more convenient to define "approximation measures" in a slightly unusual way (compare with [Ci 1]) which will be essential in certain cases. 
Let $M, N$ be positive real numbers; for convenience we assume $M \geqslant e^{e}$ (to avoid some trivialities connected with the factors $\log \log M)$, and since $15<e^{e}<16$ we assume in fact $M \geqslant 16$. Since the set $B(N, M)$ of all algebraic numbers of degree at most $N$ and measure at most $M$ is finite, in view of the trivial inequalities

$$
H(P) \leqslant\|P\| \leqslant 2^{N(P)} M(P),
$$

if $\omega$ is a transcendental number we have

$$
\min _{\xi \in B(N, M)}|\omega-\xi|>0 .
$$

Our aim is to give lower bounds for such positive numbers.

Definition. Let $\omega \in \mathbf{C}$ be a transcendental number. A real-valued function $\psi(N, \log M)$, which is defined for $N, M$ positive real numbers, with $N \geqslant 1, M \geqslant 16$, is an approximation measure for $\omega$ if

$$
|\omega-\xi| \geqslant \exp \{-\psi(N, \log M)\}
$$

for all algebraic numbers $\xi$ of degree $\leqslant N$ and measure $\leqslant M$.

In the present paper we first obtain approximation measures, and then deduce transcendence measures. To carry out this deduction we use the following lemma.

Lemma 2.3. Let $\omega \in \mathbf{C}$ be a transcendental number, and $\psi(N, \log M)$ an approximation measure for $\omega$. Assume

$$
\psi\left(N_{2}, \log M_{2}\right) \geqslant k \psi\left(N_{1}, \log M_{1}\right)
$$

for all positive integer $k$ and all real numbers $N_{1}, M_{1}, N_{2}, M_{2}$ satisfying

$$
N_{1} \geqslant 1, \quad M_{1} \geqslant 16, \quad N_{2} \geqslant k N_{1}, \quad \log M_{2} \geqslant k \log M_{1} .
$$

Then the function

$$
\psi(N, \log H+\log N)+2 N(\log H+N)
$$

is a transcendence measure for $\omega$.

This kind of result is well known (see, for example, [Ci 1] Lemmas 2.15 and 4.3), but what is important here is that we have $\psi(N, \log H+\log N)$ instead of $\psi(N, \log H+N)$. This improvement will be important, for example, for the transcendence measure of $e^{\beta}$ when $\log H<N$. 
Proof of Lemma 2.3. Let $P \in Z[X]$ be a non-trivial polynomial, and let $\xi$ be the root of $P$ which is at minimal distance from $\omega$. From a well-known result of Güting (see $[\mathrm{M}-\mathrm{W}]$ Lemma 9) we deduce

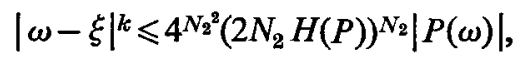

where $N_{2}=N(P)$ and $k$ is the multiplicity of the root $\xi$ of $P$.

Let $Q$ denote the minimal polynomial of $\xi$ over $\mathbf{Z}$ and let $N_{1}$ be its degree. Since $Q^{k}$ divides $P$, we have

and

$$
N_{1} \leqslant N_{2} / k
$$

$$
\log M(Q) \leqslant \frac{1}{k} \log M(P) \leqslant \frac{1}{k}\left(\log H(P)+\log N_{2}\right) .
$$

From the inequality (for $H=\max (16, H(P))$ )

$$
\log H+N_{2} \log 4+\log \left(2 N_{2}\right) \leqslant 2\left(\log H+N_{2}\right)
$$

we conclude

$$
\log |P(\omega)| \geqslant-k \psi\left(N_{1}, \log M_{1}\right)-2 N_{2}\left(\log H+N_{2}\right)
$$

with $M_{1}=\max (M(Q), 16)$. Using our assumption

$$
k \psi\left(N_{1}, \log M_{1}\right) \leqslant \psi\left(N_{2}, \log H+\log N_{2}\right)
$$

we obtain the desired result.

Finally, we give a very simple lemma which will be needed in the study of exponentials and powers.

LeMma 2.4. Let $v$ and $w$ be two complex numbers satisfying

$$
\left|w-e^{v}\right| \leqslant \frac{1}{3}\left|e^{v}\right| \text {. }
$$

then there exists a determination of the logarithm of $w$ such that

$$
\left|w-e^{v}\right| \geqslant \frac{2}{3}\left|e^{v}\right||\log w-v|
$$

Proof OF Lemma 2.4. Since the principal value of the logarithm (say $\log$ ) satisfies

$$
\sup _{|z| \leqslant \frac{1}{2}}|\log (1+z)|<\frac{1}{2},
$$

by the maximum modulus principle applied to the function $(1 / z) \log (1+z)$ we have, for $|z| \leqslant \frac{1}{3}$,

$$
|\log (1+z)| \leqslant \frac{3}{2}|z|
$$


Therefore

We define

$$
\left|\log \left(w e^{-v}\right)\right| \leqslant \frac{3}{2}\left|w e^{-v}-1\right|
$$

$$
\log w=\log \left(w e^{-v}\right)+v .
$$

This completes the proof of Lemma 2.4 .

\section{The difference between an algebraic number and the logarithm of an algebraic number}

In this part we derive several consequence of the following lower bound for $|\beta-\log \alpha|$.

THEOREM A. Let $\alpha, \beta$ be two non-zero algebraic numbers, and let $\log \alpha$ be any determination of the logarithm of $\alpha$. Let $D$ be a positive integer, and $V, E$ be positive real numbers, satisfying

and

$$
\begin{aligned}
& D \geqslant[Q(\alpha, \beta): Q], \\
& V \geqslant \max \{h(\alpha) ;|\log \alpha| / D ; 1 / D\}
\end{aligned}
$$

$$
1<E \leqslant \min \left\{e^{D V} ; 4 D V /|\log \alpha|\right\} .
$$

Finally set $V^{+}=\max \{V, 1\}$. Then

$$
|\beta-\log \alpha|>\exp \left\{-2^{35} D^{3} V\left(h(\beta)+\log \left(E D V^{+}\right)\right)(\log (E D))(\log E)^{-2}\right\} .
$$

This estimate is a special case of Theorem C, Section 5, and is proved in [W]. We show how it can be used to study the algebraic approximations of the numbers $\pi, \log \alpha$ and $e^{\beta}$.

1. Algebraic approximations to the number $\pi$

According to a remarkable result of Mahler [Ma 3,5] and an improved version of Mignotte [Mi], for every rational number $p / q$ with $q \geqslant 2$ we have

$$
\left|\pi-\frac{p}{q}\right| \geqslant q^{-21}
$$

Here we consider algebraic approximations of $\pi$. Let $\xi$ be a real algebraic number of degree $N$, with $N \geqslant 2$. Define $\alpha_{1}=-1, \log \alpha_{1}=i \pi, \beta=i \xi, D=2 N$, $V=\pi / D$ and $E=4$. From Theorem A we deduce

$$
\begin{aligned}
|\xi-\pi| & =|\beta-i \pi| \\
& \geqslant \exp \left\{-2^{35} \pi D^{2}(h(\beta)+\log D+\log 4)(\log D+\log 4) .(\log 4)^{-2}\right\} .
\end{aligned}
$$


Let $M$ satisfy $M \geqslant \max \left\{e^{N h(\xi)}, 16\right\}$. Since $h(\xi)=h(\beta)$, the inequalities

$$
\log M+N \log N+3 N \log 2 \leqslant \frac{21}{10}(\log M+N \log N)
$$

$$
\log N+3 N \log 2 \leqslant \frac{17}{10}(1+\log N)
$$

and

$$
\pi_{10}^{21} \cdot \frac{17}{10}(\log 2)^{-2}<24
$$

lead to the following result.

THEOREM 3.1. An approximation measure for $\pi$ is

$$
\text { 3.2 } 2^{38} \cdot N(\log M+N \log N)(1+\log N) \text {. }
$$

From Lemma 2.3 and the inequality

$$
\log H+N \log N+\log N \leqslant \frac{6}{5}(\log H+N \log N)
$$

(valid for $H \geqslant 16$ and $N \geqslant 2$ ) we deduce

COROLLARY 3.2. A transcendence measure for $\pi$ is

$$
2^{40} N(\log H+N \log N)(1+\log N) .
$$

The existence of an absolute constant $C_{11}>0$ such that

$$
C_{11} N(\log \mathrm{H}+N \log N)(1+\log N)
$$

is a transcendence measure for $\pi$ was first announced (and proved) by Cijouw in [Ci 5]. Actually it can be deduced from two earlier results of Fel'dman, [F 2], namely

$$
|P(\pi)|>\exp \left\{-C_{12} N(\log H+N \log N)(\log \log H+\log N)\right\}
$$

and, provided that $\log H>N^{2}(\log N)^{4}$,

$$
|P(\pi)|>\exp \left\{-C_{13} N(\log H)(1+\log N)\right\} .
$$

For if $\log H \geqslant N^{3}$, then (3.4) gives what we want, while if $\log H \leqslant N^{3}$, then (3.3) gives

$$
|P(\pi)|>\exp \left\{-3 C_{12} N(\log H+N \log N)(1+\log N)\right\}
$$

which is also what we want.

Earlier transcendence measures for $\pi$ were due to Popken (1929), Siegel (1930) and Mahler [Ma 1, 3]. (See [F-S], [Ci 1, 5] and [F 4]).

In some special cases, as already remarked by Fel'dman in 1959 (cf. F 4]), Theorem 3.1 can be improved. 
Let $m \geqslant 1$ be an integer and $\xi$ a real algebraic number; let $D_{m}$ denote the degree over $Q$ of the field $Q\left(\xi, e^{i n / m}, i\right)$. From Theorem A applied to $(i \xi / m)-(i \pi / m)$, with $\alpha=e^{i \pi / m}, \log \alpha=i \pi / m, V=(\pi+\log m) / D_{m}, E=e m$, and since

$$
h(\xi / m) \leqslant h(\xi)+\log m,
$$

we conclude

$$
\begin{aligned}
|\pi-\xi| & \geqslant\left|\frac{i \xi}{m}-\frac{i \pi}{m}\right| \\
& >\exp \left\{-2^{35} \pi D_{m}^{2}\left(h(\xi)+1+2 \log m+\log D_{m}\right)\left(1+\frac{\log D_{m}}{1+\log m}\right)\right\}
\end{aligned}
$$

(We choose $V$ rather large to enable $E$ to be large.)

THEOREM 3.5. Let $\xi$ be an algebraic number and $m$ a positive integer. Define

Then

$$
D_{m}=\left[Q\left(i, \xi, e^{i \pi / m}\right): Q\right]
$$

$$
|\pi-\xi|>\exp \left\{-2^{38} D_{m}^{2}\left(h(\xi)+\log m+\log D_{m}\right)\left(1+\frac{\log D_{m}}{1+\log m}\right)\right\}
$$

In the particular case where $\beta \in Q\left(e^{i \pi / m}\right)$, we obtain

$$
|\pi-\xi|>\exp \left\{-2^{40} D_{m}^{2}\left(h(\xi)+\log D_{m}\right)\right\},
$$

while Fel'dman obtains

$$
|\pi-\xi|>\exp \left\{-C_{14} D_{m}^{2}\left(N(\xi)^{-1} \log L(\xi)+1\right)\right\},
$$

where $C_{14}$ is an effectively computable absolute constant. In spite of the inequality $N(\xi) h(\xi) \leqslant \log L(\xi)$, Fel'dman's result is sometimes better in some cases where $m$ is large. On the other hand, our Theorem 3.5 does not assume $\beta \in Q\left(e^{i \pi / m}\right)$.

\section{Approximation of the logarithms of algebraic numbers}

Let $\alpha$ be a non-zero algebraic number and let $\log \alpha$ be a non-zero determination of the logarithm of $\alpha$. Transcendence measures for $\log \alpha$ have been proved successively by Mahler [Ma 1, 2], Fel'dman [F 1, 3] and Cijsouw [Ci 1, 2, 4]. The best known result is due to Cijsouw [Ci 4], and we give a new proof of it.

We define

$$
V=\max \{h(\alpha) ;|\log \alpha| ; 1\}, \quad d=[Q(\alpha): Q] .
$$


Let $\xi$ be an algebraic number of degree $N$ and measure at most $M$, with $M \geqslant 16$. From Theorem A with $D=N d, E=e D$, we deduce

$$
\begin{aligned}
|\log \alpha-\xi|>\exp \left\{-2^{35} \mathrm{~d}^{3} V N^{3}(\right. & \left.\frac{1}{N} \log M+2 \log N+2 \log d+1+\log V\right) \\
& \left.\times(1+2 \log N+2 \log d)(1+\log d+\log N)^{-2}\right\} .
\end{aligned}
$$

Let us define

We get:

$$
C_{15}(\alpha)=2^{38} d^{3} V(1+\log d+\log V) .
$$

THEOREM 3.6. An approximation measure for $\log \alpha$ is

$$
C_{15}(\alpha) N^{2}(\log M+N \log N)(1+\log N)^{-1} .
$$

Corollary 3.7. A transcendence measure for $\log \alpha$ is

$$
2 C_{15}(\alpha) N^{2}(\log H+N \log N)(1+\log N)^{-1} .
$$

\section{Approximation of the exponentials of algebraic numbers}

The problem of finding a transcendence measure for the number $e$ is very old (at least for the standard of the theory) since it was initiated as soon as 1899 by Borel. Later, Popken [P], Siegel (1930), Mahler (1932), [Ma 1] and Fel'dman (1963) improved the bound, and the sharpest result until now was due to Cijsouw [Ci 1,2] and was valid also for the numbers $e^{\beta}$ with $\beta$ algebraic, $\beta \neq 0$. Our result will be slightly better.

Let $\beta$ be a non-zero algebraic number and $d$ be its degree. Let $\xi$ be an algebraic number of degree $N$ and measure at most $M$, with $M \geqslant 16$, satisfying $\left|e^{\beta}-\xi\right| \leqslant \frac{1}{3}\left|e^{\beta}\right|$. From Lemma 2.4 we can find a determination $\log \xi$ of the logarithm of $\xi$ such that

$$
\left|e^{\beta}-\xi\right| \geqslant \frac{2}{3}\left|e^{\beta}\right||\beta-\log \xi| \text {. }
$$

We now use Theorem A with

$$
V=\frac{1}{N}(\log M)(1+|\beta|), \quad D=d N, \quad E=\log M .
$$

Thus

Let us define

$$
\begin{aligned}
|\beta-\log \xi| & \geqslant \exp \left\{-2^{35} d^{3} N^{2}(\log M)(1+|\beta|)\right. \\
& \times(h(\beta)+\log d+\log N+2 \log \log M+\log (1+|\beta|) \\
& \left.\times(\log d+\log \log M+\log N)(\log \log M)^{-2}\right\} .
\end{aligned}
$$

$$
C_{16}(\beta)=2^{37} d^{3}(1+|\beta|)(1+\log d)(h(\beta)+\log d+\log (1+|\beta|)+1) .
$$


THEOREM 3.8. An approximation measure for $e^{\beta}$ is

$$
\left.C_{16}(\beta) N^{2}(\log M)(\log \log M+\log N)^{2} \log \log M\right)^{-2} .
$$

If we bound $\log H+\log N$ by $\log H+N$, we deduce from Theorem 3.8 and Lemma 2.3 that

$$
4 C_{16}(\beta) N^{2}(\log H+N)
$$

is a transcendence measure for $e^{\beta}$. This is Cijsouw's result [Ci 1,2]. But, for $H \leqslant e^{N}$, we actually get a better result.

COROLLARY 3.9. A transcendence measure for $e^{\beta}$ is

$8 C_{16}(\beta) N^{2}(\log H+\log N)(\log \log H+\log N)^{2}\left(\log \log H+\log _{+} \log N\right)^{-2}$.

It is interesting to notice that the old result of Mahler [Ma 1]:

$$
\left|P\left(e^{\beta}\right)\right|>\exp \left\{-C_{17} N \log H\right\} \text { for } H \geqslant H_{0}(N)
$$

is still better for large $H$.

4. The linear form $\beta \log \alpha_{1}-\log \alpha_{2}$

We study now several consequences of the following estimate.

THEOREM B. Let $\alpha_{1}, \alpha_{2}, \beta$ be non-zero algebraic numbers, and $\log \alpha_{1}, \log \alpha_{2}$ be determinations of the logarithms of $\alpha_{1}, \alpha_{2}$ respectively. We assume

$$
\beta \log \alpha_{1} \neq \log \alpha_{2} \text {. }
$$

Let $D$ be a positive integer, and $V_{1}, V_{2}, E$ be positive real numbers, satisfying

$$
\begin{aligned}
& D \geqslant\left[Q\left(\alpha_{1}, \alpha_{2}, \beta\right): Q\right], \\
& V_{j} \geqslant \max \left\{h\left(\alpha_{j}\right),\left|\log \alpha_{j}\right| / D, 1 / D\right\} \quad(j=1,2),
\end{aligned}
$$

and

$$
1<E \leqslant \min \left\{e^{D V_{1}}, e^{D V_{2}}, 4 D V_{1} /\left|\log \alpha_{1}\right|, 4 D V_{2} /\left|\log \alpha_{2}\right|\right\} .
$$

Further define

$$
V_{j}^{+}=\max \left\{V_{j}, 1\right\} \quad(j=1,2), \quad V^{*}=\max \left\{V_{1}^{+}, V_{2}^{+}\right\}, \quad \text { and } \quad V_{*}=\min \left\{V_{1}^{+}, V_{2}^{+}\right\} .
$$

Then

$\left|\beta \log \alpha_{1}-\log \alpha_{2}\right|>\exp \left\{-2^{53} D^{4} V_{1} V_{2}\left(h(\beta)+\log \left(E D V^{*}\right)\right)\left(\log \left(E D V_{*}\right)\right)(\log E)^{-3}\right\}$. 
This result, which is proved in [W] (see Theorem C, Section V below), will be our main tool in the investigation of diophantine approximations of the numbers $\left(\log \alpha_{1}\right) /\left(\log \alpha_{2}\right)$ and $\alpha^{\beta}$. We begin with the number $e^{\pi}$.

\section{Approximation to $e^{\pi}$}

We first consider rational approximations to $e^{\pi}$. The best known result is due to Baker [B]: there exists an absolute constant $C_{18}$ such that

$$
\left|e^{\pi}-\frac{p}{q}\right|>q^{-C_{18} \log \log q}
$$

for all rational numbers $p / q$ with $q \geqslant 3$ (it is not yet known whether the extra $\log \log q$ is superfluous). We deduce from Theorem B an upper bound for $C_{18}$ as follows.

Assume first $q>\left(e^{\pi}+1\right)^{2}$ and $\left|e^{\pi}-(p / q)\right| \leqslant 1$, so that

$$
\left|\pi-\log \frac{p}{q}\right| \leqslant\left|e^{\pi}-\frac{p}{q}\right| \text { and } p<\left(e^{\pi}+1\right) q<q^{q} .
$$

We choose

$\beta=-i, \quad \alpha_{1}=-1, \quad \log \alpha_{1}=i \pi, \quad \alpha_{2}=p / q, \quad D=2, \quad V_{1}=\frac{1}{2} \pi, \quad V_{2}=\log p, \quad E=4$.

Thus

$$
\begin{aligned}
\left|e^{\pi}-\frac{p}{q}\right| & >\exp \left\{-2^{53} 2^{4} \frac{\pi}{2}(\log p)(\log 8+\log \log p)(\log 4 \pi)(\log 4)^{-3}\right\} \\
& >\exp \left\{-2^{59}(\log p)(\log \log p)\right\} \\
& >\exp \left\{-2^{60}(\log q)(\log \log q)\right\} .
\end{aligned}
$$

From the continued fraction expansion

$$
e^{\pi}=[23,7,9,3,1,1,591, \ldots]
$$

we see that these results hold also for $3 \leqslant q<583$. Therefore

$$
C_{18} \leqslant 2^{60} \text {. }
$$

We now consider algebraic approximations. Let $\xi$ be a real algebraic number with $\left|\xi-e^{\pi}\right| \leqslant 1$. The (usual) logarithm of $\xi$ satisfies, by Lemma 2.4,

$$
|\pi-\log \xi| \leqslant\left|e^{\pi}-\xi\right| \text {. }
$$

We use Theorem $B$ with

$$
D=2 N, \quad N \geqslant 2, \quad V_{1}=\pi / D, \quad V_{2}=(1 / N) \log M, \quad E=4 .
$$


We obtain

$$
\begin{array}{r}
\left|e^{\pi}-\xi\right|>\exp \left\{-2^{53} 2^{4} \frac{\pi}{2} N^{2}(\log M)(\log \log M+\log N+\log 8)\right. \\
\left.(\log N+\log 8)(\log 4)^{-3}\right\}
\end{array}
$$

THEOREM 4.1. An approximation measure for $e^{\pi}$ is

$$
2^{59} N^{2}(\log M)(\log \log M+\log N)(1+\log N) .
$$

CoROLlaRY 4.2. A transcendence measure for $e^{\pi}$ is

$$
2^{60} N^{2}(\log H+\log N)(\log \log H+\log N)(1+\log N) \text {. }
$$

Several other estimates for $e^{\pi}$ are known: Koksma and Popken [K-P], Gel'fond [G], Cijsouw [Ci 1] and Choodnovsky [Ch]. Two of them are not consequences of Corollary 4.2 , namely

$$
C_{19} N(\log H+N)^{2}(\log \log H+\log N)^{-1}
$$

(Cijsouw, "Stellingen" of [Ci 1]), and

$$
C_{20} N(\log H+\log N)^{2}(\log \log H+\log N)
$$

(Choodnovsky [Ch]), where $C_{19}, C_{20}$ are effectively computable absolute constants.

The three above-mentioned transcendence measures for $e^{\pi}$ hold uniformly $(N \geqslant 1$, $H \geqslant 16$ ), but our Corollary 4.2 is better when $\log H \geqslant N(\log N)^{3}$ (in which case we can bound $(\log H+\log N)(\log \log H+\log N)$ by $3(\log H)(\log \log H))$, Cijsouw's result is better when $N(\log N)^{3} \geqslant \log \mathrm{H} \geqslant N(\log N)^{-1}$ (and then $\log \log H+\log N \geqslant \log \log H$ does not weaken the result), while Choodnovsky's result is better when $N(\log N)^{-1} \geqslant \log H$ (which implies $\log \log H \leqslant \log N$ ). Consequently we obtain the results which we announced in Fig. 2.

We can use Theorem $B$ in the same way as we did for the proof of Theorem 3.5: let $m \geqslant 1$ be an integer; we choose

$$
\begin{aligned}
& \alpha_{1}=\xi, \quad \beta=i / m, \quad \alpha_{2}=e^{i \pi / m}, \quad \log \alpha_{2}=i \pi / m, \\
& V_{1}=h(\xi)+m(\pi-1) D_{m}^{-1}, \quad V_{2}=(\pi+\log m) D_{m}^{-1}, \quad E=e m .
\end{aligned}
$$

THEOREM 4.3. Let $\xi$ be an algebraic number and $m$ a positive integer. Denote by $D_{m}$ the degree of the field $Q\left(i, \xi, e^{i \pi / m}\right)$. Then

$$
\begin{aligned}
\left|e^{\pi}-\xi\right|>\exp \left\{-2^{60} D_{m}^{2}\left(D_{m} h(\xi)+m\right)\right. & \left(\log h(\xi)+\log D_{m}+\log m+1\right) \\
& \left.\times\left(\log D_{m}+\log m+1\right)(\log m+1)^{-2}\right\} .
\end{aligned}
$$


2. Approximation of the quotient of the logarithms of algebraic numbers

Let $\alpha_{1}, \alpha_{2}$ be two non-zero algebraic numbers, and $\log \alpha_{1}, \log \alpha_{2}$ be non-zero determinations of their logarithms. We assume that $\left(\log \alpha_{1}\right) /\left(\log \alpha_{2}\right)$ is irrational. Transcendence measures for this number are due to Gel'fond $(1935,1939,1949)$ (see [F-S] and [G]) and to Cijsouw [Ci 1]. Our Theorem B leads to a sharpening of these measures. Let us define

and

$$
d=\left[Q\left(\alpha_{1}, \alpha_{2}\right): Q\right], \quad V_{j}=1+h\left(\alpha_{j}\right)+\left|\log \alpha_{j}\right|, \quad(j=1,2),
$$

$$
C_{21}\left(\alpha_{1}, \alpha_{2}\right)=2^{55} d^{4} V_{1} V_{2}\left(1+\log V_{1}\right)\left(1+\log V_{2}\right) .
$$

We choose $D=d N, E=e D$.

THEOREM 4.4. An approximation measure for $\left(\log \alpha_{1}\right) /\left(\log \alpha_{2}\right)$ is

$$
C_{21}\left(\alpha_{1}, \alpha_{2}\right) N^{3}(\log M+N \log N)(1+\log N)^{-2} .
$$

COROLlary 4.5. A transcendence measure for $\left(\log \alpha_{1}\right) /\left(\log \alpha_{2}\right)$ is

$$
2 C_{21}\left(\alpha_{1}, \alpha_{2}\right) N^{3}(\log H+N \log N)(1+\log N)^{-2} .
$$

3. Approximation of algebraic powers of algebraic numbers

Let $\alpha$ and $\beta$ be non-zero algebraic numbers, with $\beta$ irrational, and let $\log \alpha$ be a non-zero determination of the logarithm of $\alpha$. Define as usual $\alpha^{\beta}=\exp (\beta \log \alpha)$. Further let

$$
d=[Q(\alpha, \beta): Q], \quad V=1+h(\alpha)+|\log \alpha| .
$$

Let $\xi$ be an algebraic number of degree $N$ and measure at most $M$, with $M \geqslant 16$, such that

$$
\left|\alpha^{\beta}-\xi\right| \leqslant \frac{1}{3}\left|\alpha^{\beta}\right| \text {. }
$$

By Lemma 2.4 we can choose a determination $\log \xi$ of the logarithm of $\ddot{\xi}$ such that

$$
\left|\alpha^{\beta}-\xi\right| \geqslant \frac{2}{3}\left|\alpha^{\beta}\right| \cdot|\beta \log \alpha-\log \xi| \text {. }
$$

We now choose

$$
D=N d, \quad V_{1}=V, \quad V_{2}=\frac{1}{N}(\log M)(1+|\beta \log \alpha|),
$$

and

Finally define

$$
E=\min \{e N, \log M\}
$$

$$
\begin{aligned}
C_{22}(\beta, \log \alpha) & =6.2^{53} d^{4} V(1+|\beta \log \alpha|) \\
& (1+h(\beta)+\log V+\log d+|\beta \log \alpha|)(1+\log V+\log d) .
\end{aligned}
$$


Then Theorem $\mathbf{B}$ gives

$$
\begin{aligned}
\left|\alpha^{\beta}-\xi\right|>\exp \left\{-\frac{1}{6} C_{22}(\beta, \log \alpha) N^{3}(\log M)(\log \right. & \log M+\log N+\log E) \\
& \left.\times(\log N+\log E)(\log E)^{-3}\right\} .
\end{aligned}
$$

In the case $\log M \geqslant e N$ we get

$$
\left|\alpha^{\beta}-\xi\right|>\exp \left\{-C_{22}(\beta, \log \alpha) N^{3}(\log M)(\log \log M)(1+\log N)^{-2}\right\} .
$$

While in the case $\log M \leqslant e N$ we obtain

$$
\left|\alpha^{\beta}-\xi\right|>\exp \left\{-C_{22}(\beta, \log \alpha) N^{3}(\log M)(1+\log N)^{2}(\log \log M)^{-3}\right\} .
$$

We conclude

THEOREM 4.6. An approximation measure for $\alpha^{\beta}$ is

$$
C_{22}(\beta, \log \alpha) N^{3}(\log M)(\log \log M+\log N)^{4}(\log \log M)^{-3}(1+\log N)^{-2} .
$$

From Lemma 2.3 we deduce that

$$
\begin{aligned}
2^{4} C_{22}(\beta, \log \alpha) N^{3}(\log H+ & \log N)(\log \log H+\log N)^{4} \\
& \times\left(\log \log H+\log _{+} \log N\right)^{-3}(1+\log N)^{-2}
\end{aligned}
$$

is a transcendence measure for $\alpha^{\beta}$. This result can be improved in the following way. If $\log H>N$, this transcendence measure is less than

$$
2^{9} C_{22}(\beta, \log \alpha) N^{3}(\log H)(\log \log H)(1+\log N)^{-2} .
$$

On the other hand, we know by [Ch] Theorem 1.5 that there exists a constant $C_{23}(\beta, \log \alpha)$ such that

$$
C_{23}(\beta, \log \alpha) N^{3}(\log H+\log N)(\log \log H+\log N)^{2}(1+\log N)^{-3}
$$

is a transcendence measure for $\alpha^{\beta}$. When $\log H \leqslant N$, this measure is less than

$$
4 C_{23}(\beta, \log \alpha) N^{3}(\log H+\log N)(1+\log N)^{-1} .
$$

These two estimates enable us to conclude

THEOREM 4.7. There exists a positive real number $C_{24}(\beta, \log \alpha)$ such that

$$
C_{24}(\beta, \log \alpha) N^{3}(\log H+\log N)(\log \log H+\log N)(1+\log N)^{-2}
$$

is a transcendence measure for $\alpha^{\beta}$.

This measure improves earlier results of Gel'fond (see [G], [F-S], [(Ci 1]). In the special case where $\beta$ is quadratic, Sidlovskii proved in 1951 a result which is sharper for large $N$ (see $[\mathrm{F}-\mathrm{S}]$ ). 


\section{Two general transcendence measures}

In this last part we give two results, one concerning the quotient of linear forms in logarithms and the other concerning $e^{\beta_{0}} \alpha_{1}^{\beta_{1}} \ldots \alpha_{m}^{\beta_{m}}$. Our main tool is the following estimate which is proved in [W]. (For earlier estimates we refer to the paper of Baker in the same volume.)

THEOREM C. Let $\alpha_{1}, \ldots, \alpha_{n}$ be non-zero algebraic numbers, and $\beta_{0}, \beta_{1}, \ldots, \beta_{n}$ be algebraic numbers. For $1 \leqslant j \leqslant n$, let $\log \alpha_{j}$ be any determination of the logarithm of $\alpha_{j}$. Let $D$ be a positive integer, and $V_{1}, \ldots, V_{n} W, E$ be positive real numbers, satisfying

and

$$
\begin{aligned}
& D \geqslant\left[Q\left(\alpha_{1}, \ldots, \alpha_{n}, \beta_{0}, \ldots, \beta_{n}\right): Q\right], \\
& V_{j} \geqslant \max \left\{h\left(\alpha_{j}\right),\left|\log \alpha_{j}\right| / D, 1 / D\right\} \quad(1 \leqslant j \leqslant n), \\
& W \geqslant \max _{0 \leqslant j \leqslant n}\left\{h\left(\beta_{j}\right)\right\}, \\
& V_{1} \leqslant \ldots \leqslant V_{n}
\end{aligned}
$$

$$
1<E \leqslant \min \left\{e^{D V_{1}} ; \min _{1 \leqslant j \leqslant n} 4 D V_{j} /\left|\log \alpha_{j}\right| .\right.
$$

Finally define $V_{j}^{+}=\max \left\{V_{j}, 1\right\}$ for $j=n$ and $j=n-1$, with $V_{0}^{+}=1$ in the case $n=1$. If the number

$$
\Lambda=\beta_{0}+\beta_{1} \log \alpha_{1}+\ldots+\beta_{n} \log \alpha_{n}
$$

does not vanish, then

$$
\left.|\Lambda|>\exp \left\{-C(n) D^{n+2} V_{1} \ldots V_{n}\left(W+\log \left(E D V_{n}^{+}\right)\right)\left(\log E D V_{n-1}^{+}\right)\right)(\log E)^{-n-1}\right\},
$$

where

$$
C(1) \leqslant 2^{35}, \quad C(2) \leqslant 2^{53} \text { and } \quad C(n) \leqslant 2^{8 n+51} n^{2 n} .
$$

\section{Quotient of linear forms in logarithms}

Let $\alpha_{1}, \ldots, \alpha_{g}, \alpha_{1}^{\prime}, \ldots, \alpha_{t}^{\prime}$ be non-zero algebraic numbers, $\beta_{0}, \ldots, \beta_{s}, \beta_{0}^{\prime}, \ldots, \beta_{t}^{\prime}$ be algebraic numbers. For $1 \leqslant j \leqslant s\left(\right.$ resp. $1 \leqslant k \leqslant t$ ) let $\log \alpha_{j}$ (resp. $\log \alpha_{k}^{\prime}$ ) be a determination of the logarithm of $\alpha_{j}$ (resp. of $\alpha_{k}^{\prime}$ ). We assume that

$$
\beta_{0}^{\prime}+\beta_{1}^{\prime} \log \alpha_{1}^{\prime}+\ldots+\beta_{t}^{\prime} \log \alpha_{t}^{\prime}
$$

does not vanish, and that the number

$$
\omega=\frac{\beta_{0}+\beta_{1} \log \alpha_{1}+\ldots+\beta_{s} \log \alpha_{s}}{\beta_{0}^{\prime}+\beta_{1}^{\prime} \log \alpha_{1}^{\prime}+\ldots+\beta_{t}^{\prime} \log \alpha_{t}^{\prime}}
$$

is transcendental. (Thanks to Baker [B] Chapter 3 we know when this assumption is fullfilled.) We give here an estimate for the approximation of $\omega$ which improves substantially previous results of Cijsouw [Ci 1, 3]. 
THEOREM 5.1. There exists an easily computable constant $C_{25}$, depending only on $\log \alpha_{1}, \ldots, \log \alpha_{s}, \log \alpha_{1}^{\prime}, \ldots, \log \alpha_{i}^{\prime}, \beta_{0}, \ldots, \beta_{s}, \beta_{0}^{\prime}, \ldots, \beta_{l}^{\prime}$, with the following property.

1. Let $r_{1}+1$ be the dimension of the $Q$-vector space generated by $\log \alpha_{1}, \ldots, \log \alpha_{s}$, $\log \alpha_{1}^{\prime}, \ldots, \log \alpha_{t}^{\prime}, i \pi$. Then

$$
C_{25} N^{r_{1}+1}(\log M+N \log N)(1+\log N)
$$

is an approximation measure for $\omega$, and consequently

$$
2 C_{25} N^{r_{1}+1}(\log H+N \log N)(1+\log N)
$$

is a transcendence measure for $\omega$.

2. Let $r_{2}$ be the dimension of the $Q$-vector space generated by $\log \alpha_{1}, \ldots, \log \alpha_{s}$, $\log \alpha_{1}^{\prime}, \ldots, \log \alpha_{t^{\prime}}^{\prime}$ Then

$$
C_{25} N^{r_{2}+1}(\log M+N \log N)(1+\log N)^{-r_{2}}
$$

is an approximation measure for $\omega$, and consequently

$$
2 C_{25} N^{r_{2}+1}(\log H+N \log N)(1+\log N)^{-r_{2}}
$$

is a transcendence measure for $\omega$.

Plainly we have $r_{1}=r_{2}$ or $r_{1}=r_{2}-1$. If $r_{1}=r_{2}$ the second result is slightly sharper, while if $r_{1}=r_{2}-1$ (which means that $i \pi$ is a linear combination of $\log \alpha_{1}, \ldots, \log \alpha_{t}^{\prime}$ with rational coefficients) the first estimate is quite sharp.

From Theorem 5.1 it follows that the transcendence type of $\omega$ is at most $r_{1}+2+\varepsilon$ for all $\varepsilon>0$ and at most $r_{2}+2$.

It is readily seen that Theorem 5.1 contains the above-mentioned transcendence measures for $\pi$ (with $r_{1}=0$; cf. (3.2)), for $\log \alpha$ (with $r_{2}=1$; cf. (3.7)) and for $\left(\log \alpha_{1}\right) /\left(\log \alpha_{2}\right)$ (with $r_{2}=2$; cf. (4.5)). Here is another consequence corresponding to $r_{1}=1$.

COROLLARY 5.2. Let $\log \alpha$ be a logarithm of an algebraic number, and let $\beta_{0}, \beta_{1}$, $\beta_{0}^{\prime}, \beta_{1}^{\prime}, \gamma, \gamma^{\prime}$ be algebraic numbers such that the number

$$
\beta_{0}^{\prime}+\beta_{1}^{\prime} \log \alpha+\gamma^{\prime} i \pi
$$

does not vanish, and that the number

$$
\frac{\beta_{0}+\beta_{1} \log \alpha+\gamma i \pi}{\beta_{0}^{\prime}+\beta_{1}^{\prime} \log \alpha+\gamma^{\prime} i \pi}
$$

is transcendental. Then this number has a transcendence measure

$$
C_{26} N^{2}(\log H+N \log N)(1+\log N),
$$

where $C_{26}$ depends only on $\log \alpha, \beta_{0}, \beta_{1}, \beta_{0}^{\prime}, \beta_{1}^{\prime}, \gamma, \gamma^{\prime}$.

Therefore this number has a transcendence type at most $3+\varepsilon$ for all $\varepsilon>0$. 
Proof of Theorem 5.1. Let $\log a_{1}, \ldots, \log a_{r_{1}+1}$ be a basis of the $Q$-vector space generated by $\log \alpha_{1}, \ldots, \log \alpha_{s}, \log \alpha_{1}^{\prime}, \ldots, \log \alpha_{t}^{\prime}, i \pi$, with $\log a_{1}=i \pi$. Thus there exist rational numbers $b_{j, l}, b_{k, l}^{\prime}\left(1 \leqslant j \leqslant s, 1 \leqslant k \leqslant t, 1 \leqslant l \leqslant r_{1}+1\right)$ such that

$$
\log \alpha_{j}=\sum_{l=1}^{r_{1}+1} b_{j,} \log a_{l} \quad(1 \leqslant j \leqslant s),
$$

and

$$
\log \alpha_{k}^{\prime}=\sum_{l=1}^{r_{1}+1} b_{k, l}^{\prime} \log a_{l} \quad(1 \leqslant k \leqslant t) .
$$

Let $\xi$ be an algebraic number of degree $N$ and measure at most $M$, with $M \geqslant 16$. Then

where

$$
0<|\omega-\xi|=|\Lambda| .\left|\beta_{0}^{\prime}+\beta_{1}^{\prime} \log \alpha_{1}^{\prime}+\ldots+\beta_{t}^{\prime} \log \alpha_{t}^{\prime}\right|,
$$

$$
\begin{aligned}
\Lambda & =\beta_{0}+\sum_{j=1}^{s} \beta_{j} \log \alpha_{j}-\xi \beta_{0}^{\prime}-\xi \sum_{k=1}^{t} \beta_{t}^{\prime} \log \alpha_{k}^{\prime} \\
& =\left(\beta_{0}-\xi \beta_{0}^{\prime}\right)+\sum_{l=1}^{r_{1}+1}\left(\sum_{j=1}^{s} \beta_{j} b_{j, l}-\xi \sum_{k=1}^{t} \beta_{k}^{\prime} b_{k, l}^{\prime}\right) \log a_{l} .
\end{aligned}
$$

Theorem $\mathrm{C}$ provides us a lower bound for $|\Lambda|$, with

$$
n=r_{1}+1, \quad W=C_{27}(1 / N) \log M, \quad V_{1}=1 / D, \quad D=C_{28} N, \quad E=4
$$

and $C_{27}, C_{28}, V_{2}, \ldots, V_{n}$ are easily computable constants (for instance

$$
\left.C_{28}=\left[Q\left(\beta_{0}, \ldots, \beta_{t}^{\prime}, \ldots, \alpha_{t}^{\prime}\right)\right]\right) .
$$

Now let $\log a_{1}^{\prime}, \ldots, \log a_{r_{2}}^{\prime}$ be a basis of the $Q$ vector space generated by $\log \alpha_{1}, \ldots, \log \alpha_{t}^{\prime}$. Using the previous arguments, we need a lower bound for

$$
\Lambda=\beta_{0}-\xi \beta_{0}^{\prime}+\sum_{l=1}^{r_{2}} \gamma_{l} \log a_{l}^{\prime}
$$

where $\gamma_{l}$ is a linear combination of 1 and $\xi$ with fixed coefficients in $Q\left(\beta_{0}, \ldots, \beta_{l}^{\prime}\right)$. We now choose $n=r_{2}, E=e N$, while $V_{1}, \ldots, V_{n}$ are constants. The desired result plainly follows from Theorem $C$. (The non-vanishing of $\Lambda$ is a consequence of the transcendence of $\omega$.)

\section{Product of algebraic powers of algebraic numbers}

Let $\alpha_{1}, \ldots, \alpha_{m}$ be non-zero algebraic numbers, $\beta_{0}, \ldots, \beta_{m}$ be algebraic numbers, and, for $1 \leqslant j \leqslant m$, let $\log \alpha_{j}$ be any determination of the logarithm of $\alpha_{j}$. We assume that the number

$$
\theta=\exp \left(\beta_{0}+\sum_{j=1}^{m} \beta_{j} \log \alpha_{j}\right)
$$


which we write for shortness

$$
\theta=e^{\beta_{0}} \alpha_{1}^{\beta_{1}} \ldots \alpha_{m}^{\beta_{m}},
$$

is transcendental (once more we refer to [B] to see which are the only trivial circumstances where such a number could be algebraic).

THEOREM 5.3. There exists an easily computable constant $C_{29}$, depending only on $\log \alpha_{1}, \ldots, \log \alpha_{m}, \beta_{0}, \ldots, \beta_{m}$, such that the following holds.

1. Denote by $r_{1}+1$ the dimension of the $Q$-vector space generated by $\log \alpha_{1}, \ldots, \log \alpha_{m}, i \pi$. Then $C_{29} N^{r_{1}+2}(\log M)(\log \log M+\log N)(1+\log N)$ is an approximation measure for $\theta$. Therefore

$$
2 C_{29} N^{r_{1}+2}(\log H+\log N)(\log \log H+\log N)(1+\log N)
$$

is a transcendence measure for $\theta$.

2. Denote by $r_{2}$ the dimension of the Q-vector space generated by $\log \alpha_{1}, \ldots, \log \alpha_{m}$. Then

$$
C_{29} N^{r_{2}+2}(\log M)(\log \log M+\log N)^{r_{2}+3}(\log \log M)^{-r_{2}-2}(1+\log N)^{-r_{2}-1}
$$

is an approximation measure for $\theta$, and therefore

$$
2 C_{29} N^{r_{2}+2}(\log H+\log N)(\log \log H+\log N)^{r_{2}+3}
$$

$$
(\log \log H)^{-r_{2}-2}(1+\log N)^{-r_{2}-1}
$$

is a transcendence measure for $\theta$.

Again the first estimate is much more interesting if $r_{1}=r_{2}-1$ and the second if $r_{1}=r_{2}$.

The transcendence type of $\theta$ is at most $r_{1}+3+\varepsilon$ for all $\varepsilon>0$, and at most $r_{2}+3$.

Theorem 5.3 generalizes our approximation measure for $\alpha^{\beta}$ (with $r_{2}=1$; cf. (4.6)) and our transcendence measure for $e^{\pi}$ (with $r_{1}=0$; cf. (4.2)). Indeed, the case $r_{1}=0$ shows that the transcendence measure (4.2) of the number $e^{\pi}$ holds also for the numbers $e^{\beta+\gamma \pi}$ with $\beta$ and $\gamma$ algebraic.

Earlier transcendence measures for $\theta$ were due to Cijsouw [Ci 1, 3]. In the case of bounded degree, a partial result of Smelev (1969) concerning the case $m=1$ has been improved and extended to the general case by Baker [B] Chapter 3. All these results follow from Theorem 5.3.

Proof OF THEOREM 5.3. Let $\xi$ be an algebraic number with $|\xi-\theta|<|\theta| / 3$. Using Lemma 2.4, we reduce the problem to a lower bound of

$$
\beta_{0}+\gamma_{1} \log \alpha_{1}+\ldots+\gamma_{n-1} \log \alpha_{n-1}-\log \xi
$$


with $n=r_{1}+2$ and $\log \alpha_{1}=i \pi$ in the first case, $n=r_{2}+1$ in the second case; $\gamma_{1}, \ldots, \gamma_{n}$ are fixed algebraic numbers.

We use Theorem $\mathrm{C}$ with $V_{n}=C_{30}(1 / N)(\log M)$, and

$$
V_{2}, \ldots, V_{n-1}, \quad W \text { fixed, } V_{1}=1 / N \text {, and } E=4
$$

in the first case,

$$
V_{1}, V_{2}, \ldots, V_{n-1}, \quad W \text { fixed, and } E=\min \{e N, \log M\}
$$

in the second case.

This completes the proof of Theorem 5.3.

\section{A final remark}

It is rather surprising that so few results are not consequences of our lower bound [W]. However, it seems worthwhile to go on in this field by looking at special cases, like $\pi$ (cf. [F 4]), or $e^{\pi}$ (cf. [Ch] in the case of large degree) and to use the specific properties of these numbers to improve the known results. In fact, the sharpest known results are far from best possible (namely $C_{31} N \log H$ for the transcendence measures) and there is still a lot of work to do.

From the continued fraction expansions of $\pi$ and $e^{\pi}$ up to 80 places, which were kindly provided to me by David Hunt, it follows that the only exceptions to

$$
\left|\pi-\frac{p}{q}\right|>q^{-3}
$$

in the range $2 \leqslant q \leqslant 10^{41}$ are for $q=7$ and $q=113$, and similarly the only exceptions to

$$
\left|e^{\pi}-\frac{p}{q}\right|>q^{-3}
$$

in the range $2 \leqslant q \leqslant 10^{44}$ are for $q=7$ and $q=462$.

\section{References}

[B] A. Baker (1975), Transcendental Number Theory (Cambridge University Press).

[Ch] G. V. Cudnovskii (1974), "The Gelfond-Baker method in problems of diophantine approximation", Colloquia Math. Soc. Janos Bolyai 13, 19-30 (North Holland, Amsterdam, 1975).

[Ci 1] P. L. Cijsouw (1972), Transcendence Measures (Acad. Proefschrift, Amsterdam).

[Ci 2] P. L. Cijsouw (1974), "Transcendence measures of exponentials and logarithms of algebraic numbers", Compositio Math. 28, 163-178.

[Ci 3] P. L. Cijsouw (1974), "Transcendence measures of certain numbers whose transcendency was proved by A. Baker", Compositio Math. 28, 179-194.

[Ci 4] P. L. Cijsouw (1974/5), "On the approximability of the logarithms of algebraic numbers", Sém. DELANGE-PISOT-POITOU (Théorie des Nombres), 16e année no. 19. 
[Ci 5] P. L. Cijsouw (1977), “A transcendence measure for $\pi$ ", Transcendence Theory, Advances and Applications, edited by A. Baker and D. W. Masser (Academic Press, New York).

[C-W] P. L. Cijsouw and M. Waldschmidt (1977), "Linear forms and simultaneous approximations", Compositio Math. 34, 173-197.

[F1] N. I. Fel'dman (1966), "Approximation of certain transcendental numbers. I. The approximation of logarithms of algebraic numbers", English transl., Amer. Math. Soc. Transl. II ser., 59, 224-245.

[F 2] N. I. Fel'dman (1966), "On the measure of transcendence of $\pi$ ", English transl., Amer. Math. Soc. Transl. II ser., 58, 110-124.

[F 3] Fel'dman (1966), "Approximation of the logarithms of algebraic numbers by algebraic numbers", English transl., Amer. Math. Soc. Transl. II ser., 58, 125-142.

[F 4] N. I. Fel'dman (1977), "Approximation of number $\pi$ by algebraic numbers from special fields", J. Number Theory 9, 48-60.

[F-S] N. I. Fel'dman and A. B. Šidlovskii (1967), "The development and present state of the theory of transcendental numbers", English transl., Russian Math. Surveys, 22, 1-79.

[G] A. O. Gel'fond (1960), Transcendental and Algebraic Numbers, English transl. (Dover Publications, New York).

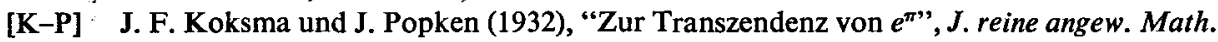
168, 211-230.

[Ma 1] K. Mahler (1932), "Zur Approximation der Exponentialfunktion und des Logarithmus", J. reine angew. Math. 166, 118-150.

[Ma 2] K. Mahler (1953), "On the approximation of logarithms of algebraic numbers", Philos. Trans. Roy. Soc. London, Ser. A, 245, 371-398.

[Ma 3] K. Mahler (1953), "On the approximation of $\pi$ ", Nederl. Akad. Wetensch. Proc., Ser. A, 56 (= Indag. Math. 15), 30-42.

[Ma 4] Mahler (1962), “On some inqualities for polynomials in several variables", J. London Math. Soc. 37, 341-344.

Ma 5] K. Mahler (1967), "Application of some formulae by Hermite to the approximation of exponentials and logarithms", Math. Ann. 168, 200-227.

[Mi] M. Mignotte (1974), "Approximations rationnelles de $\pi$ et quelques autres nombres", Journées Arithm. [1973, Grenoble], Bull. Soc. Math. France, Mémoire 37, 121-132.

[M-W] M. Mignotte and M. Waldschmidt (1978), "Linear forms in two logarithms and Schneider's method", Math. Annalen. 231, 241-267.

[P] J. Popken (1929) "Zur Transzendenz von $e$ ", Math. Zeitscht. 29, 525-541.

[W] M. Waldschmidt (to appear), "A lower bound for linear forms in logarithms", Acta Arith. 37 (1979).

Université P. et M. Curie (Paris VI)

Mathématiques, T. 45-46

4, Place Jussieu

75230 Paris Cedex 05

France 